\title{
Children (and Adults) Benefit From Visual Feedback during Gesture Interaction on Mobile Touchscreen Devices
}

\author{
Lisa Anthony ${ }^{+, 1}$, Quincy Brown ${ }^{++}$, Jaye Nias ${ }^{++}$, Berthel Tate ${ }^{++}$ \\ + UMBC \\ Information Systems \\ 1000 Hilltop Circle \\ Baltimore MD 21250 USA \\ lanthony@umbc.edu \\ ${ }^{++}$Bowie State University \\ Computer Science Department \\ 14000 Jericho Park Road \\ Bowie MD 20715 USA \\ qbrown@bowiestate.edu
}

\begin{abstract}
Surface gesture interaction styles used on mobile touchscreen devices often depend on the platform and application. Some applications show a visual trace of gesture input being made by the user, whereas others do not. Little work has been done examining the usability of visual feedback for surface gestures, especially for children. In this paper, we extend our previous work on an empirical study conducted with children, teens, and adults to explore characteristics of gesture interaction with and without visual feedback. We analyze 9 simple and 7 complex gesture features to determine whether differences exist between users of different age groups when completing surface gestures with and without visual feedback. We find that the gestures generated diverge significantly in ways that make them difficult to interpret by some recognizers. For example, users tend to make gestures with fewer strokes in the absence of visual feedback, and tend to make shorter, more compact gestures using straighter lines in the presence of visual feedback. In addition, users prefer to see visual feedback. Based on these findings, we present design recommendations for surface gesture interfaces for children, teens, and adults on mobile touchscreen devices. We recommend providing visual feedback, especially for children, wherever possible.
\end{abstract}

\section{Keywords}

Gesture interaction; surface gestures; interaction design; empirical studies; mobile devices; child-computer interaction.

\section{INTRODUCTION}

Touch interaction on mobile devices such as smartphones and tablet computers has become one of the most prevalent modes of interaction with technology for many users. These devices all support some form of surface gesture interaction, but the specific interaction styles used are often dependent on the platform and application (app). While some gestures have emerged as cross-platform standards, such as swipe, pinch-to-zoom, and dragto-pan, there is still quite a variety of other gestures in use for specific apps. For example, the note-taking and

\footnotetext{
${ }^{1}$ Corresponding author.
} 
sketching app from FiftyThree, Inc., called Paper², uses a counter-clockwise spiral gesture to "rewind" (e.g., undo) the user's command history. Another example is Realmac Software's Clear ${ }^{3}$ list-keeping app, which uses a drag-and-hold gesture to create a new list item. Anthony et al. (Anthony, Vatavu, \& Wobbrock, 2013) and Zhai et al. (Zhai, Kristensson, Appert, Anderson, \& Cao, 2012) both include summaries of the range of gestures used in research on surface gesture interaction, some of which are also used in commercial apps. In addition to using a variety of gestures, some applications show a visual trace of gesture input as it is made by the user, such as drawing or tracing games (e.g., Luck-u's Art Penguin ${ }^{4}$ ), whereas others do not, such as navigation apps (e.g., Ulmon GmbH's City Maps $2 \mathrm{Go}^{5}$ ). Evidence from cognitive and perceptual psychology literature suggests that both children and adults have more difficulty drawing and writing in the absence of visual feedback (De Bruyn \& Davis, 2008; Smyth, 1989; Vinter \& Meulenbroek, 1993). Young children may benefit even more strongly from the use of visual feedback during interaction because they are still developing the required sensorimotor coordination ability to draw without looking (Vinter \& Meulenbroek, 1993). However, little work has been done to examine the usability of visual feedback for surface gestures in general, let alone for children or teens.

In this paper, we extend previously presented results from an empirical study conducted with 41 children, teens, and adults to explore characteristics of gesture interaction with and without visual feedback (Anthony, Brown, Nias, \& Tate, 2013). We asked questions such as: How well are children, teens, and adults able to enter surface gestures with and without visual feedback? Does presence or absence of visual feedback affect consistency of the gestures made? If so, is automatic gesture recognition impacted by these changes in consistency? Which mode of gesture input (with or without feedback) do adults, teens, and children prefer?
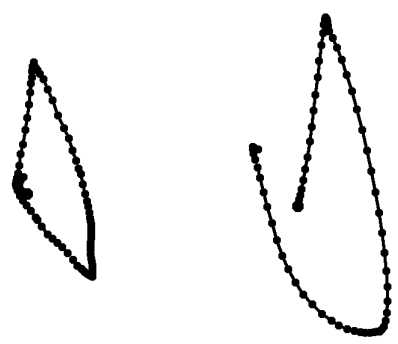

\section{$\begin{array}{ll}\text { (a) with feedback } & \text { (b) without feedback }\end{array}$ \\ Figure 1. Examples of gestures (diamond shape) produced with and without visual feedback by one participant (a child, to scale).}

We find that the gestures generated by users of different ages with and without visual feedback diverge significantly in ways that make them difficult to interpret (Figure 1). For example, users tend to make gestures with fewer strokes in the absence of visual feedback. They also tend to make shorter, more compact gestures using straighter lines with more efficiency and less wobbling in the presence of visual feedback. In addition, based on our observations, users of all age groups we studied prefer to see visual feedback, although adults are

\footnotetext{
${ }^{2}$ https://itunes.apple.com/us/app/paper-by-fiftythree/id506003812

${ }^{3}$ https://itunes.apple.com/us/app/id493136154

${ }^{4}$ https://itunes.apple.com/us/app/id449097181

${ }^{5}$ https://itunes.apple.com/us/app/city-maps-2go/id327783342
} 
more willing to accept lack of feedback. Based on these findings, we present several design recommendations for new surface gesture interfaces for children, teens, and adults on mobile touchscreen devices.

The contributions of this work include the following. First, we present an analysis of gesture features that change when visual feedback is present or absent during the interaction, which can be used to design better gesture sets and recognizers for one or both situations. Second, we analyze the actual impact of these feature differences on recognition by current algorithms used in user interaction research. Third, we present design recommendations based on empirical data we collected from children, teens, and adults related to the necessity, utility, and desirability of visual feedback. We go beyond prior presentations of this work (Anthony, Brown, et al., 2013) by investigating additional features, testing with a new recognizer, and adding to the design recommendations. The results of this work are informative to designers and researchers interested in surface gesture interaction on mobile devices for users of all ages.

\section{RELATED WORK}

We briefly survey related work on surface gesture interaction on mobile devices for both children and adults, as well as prior work on usability of interactions with and without visual feedback.

\subsection{Surface Gestures and Mobile Devices}

Gesture-based interaction on touch-enabled surfaces have been studied extensively in the $\mathrm{HCl}$ literature, particularly from a usability perspective (Frisch, Heydekorn, \& Dachselt, 2009; Harris et al., 2009; Kammer, Wojdziak, Keck, Groh, \& Taranko, 2010; Kane, Wobbrock, \& Ladner, 2011; Morris, Wobbrock, \& Wilson, 2010; Rick et al., 2009; Ryall, Morris, Everitt, Forlines, \& Shen, 2006; Tu, Ren, \& Zhai, 2012; Wobbrock, Morris, \& Wilson, 2009). Gesture set design (Morris et al., 2010; Wobbrock et al., 2009), multitouch gestures (Frisch et al., 2009; Kammer et al., 2010), accessible gestures (Kane et al., 2011), and differences between pen/stylus and finger gesture input (Tu et al., 2012) are just some of the areas that have been examined, but none of these studies have included children. From a child-computer interaction perspective, surface gestures for children especially on mobile devices have generally been neglected. Multitouch gestures for children on tabletops have been explored (Harris et al., 2009; Rick et al., 2009; Ryall et al., 2006), but research typically has either included children only, or has not distinguished between adults and children, making the comparisons needed for tailored interaction design difficult. Some work recently has explicitly compared and contrasted surface-gesture interaction design for children and adults (Anthony, Brown, Nias, Tate, \& Mohan, 2012; Brown \& Anthony, 2012; Hinrichs \& Carpendale, 2011), but has not specifically looked at the question of feedback. As we continue to see an increase in the use of touch-based mobile technologies by children (Shuler, 2009), further work in this area is needed.

Related work in pen-based handwriting interactions for children (Read, MacFarlane, \& Casey, 2002), pointing and mouse pathing interactions for children (Donker \& Reitsma, 2007; Hourcade, Bederson, Druin, \& Guimbretière, 2004; Jones, 1991; Rösblad, 2006), and drag-and-drop gestures (with mice or fingers) for children (Brown, Hatley, Bonsignore, \& Druin, 2011; Inkpen, 2001; Joiner, Messer, Light, \& Littleton, 1998) have found that children make less stable movements, have difficulty maintaining contact with the screen, and make more input errors overall than do adults. We predict that similar results will hold for other types of surface gestures performed on mobile touchscreen devices, such as the ones we study in this paper, and we explore this relationship in our own work. 


\subsection{Usability and Visual Feedback}

Past researchers have examined the use of visual feedback (among other types of feedback) for various modalities such as pointing with a mouse (Akamatsu, MacKenzie, \& Hasbroucq, 1995), text entry (Clawson, Lyons, Starner, \& Clarkson, 2005), 3D gestures (Kratz \& Ballagas, 2009), and hand-tracking gestures (Lin, Cassidy, Hook, \& Baliga, 2002). In these cases, visual feedback is usually found to be necessary to allow users to understand that their input has had the desired effect. In Clawson et al.'s work (Clawson et al., 2005), however, the visual feedback that was preferred by users during mobile text entry had the side effect of decreasing typing speed, because visible input errors distracted users. Two examples of work that explicitly seeks to reduce reliance on visual feedback are Gustafson's (Gustafson, 2012) "imaginary interfaces," which uses accelerometerbased gestures on screen-less devices, and Zhao et al.'s (Zhao, Dragicevic, Chignell, Balakrishnan, \& Baudisch, 2007) EarPod, an eyes-free menu selection technique that uses auditory rather than visual feedback. In both cases, the benefit of eyes-free interaction trades off with a new burden on the user to recall required input actions without visual confirmation of their successful interaction.

Very little work has explored the use of visual feedback for touch and gesture interaction. One example is Li's (Li, 2010) GestureSearch tool, which accepts letter gestures as shortcuts for searching, e.g., to jump to a particular alphabetic section of one's contact list. In that work, users prefer character-based gesture shortcuts for commands due to the mode switch required by text entry on mobile devices. Gesture interaction differs from other modalities in that it can support two types of visual feedback: visual feedback of the actual action being entered (e.g., the trace of a gesture), and visual feedback of the action's effect (e.g., the recognition of a gesture). Work on visual feedback in other modalities can provide design recommendations for the latter type of visual feedback. We are the first to examine the former type.

In addition, none of these studies in any modality has involved child users. Based on child development literature (e.g., (Vinter \& Meulenbroek, 1993)), we hypothesize that providing visual feedback will be even more

\begin{tabular}{|l|l|l|l|}
\hline & Overall & $\begin{array}{l}\text { Children / } \\
\text { Teens }\end{array}$ & Adults \\
\hline $\mathbf{N}$ & 41 & 25 & 16 \\
\hline Gender & 20 female & $\begin{array}{l}14 \text { female } \\
(56 \%)\end{array}$ & $\begin{array}{l}6 \text { female } \\
(38 \%)\end{array}$ \\
\hline Age (yrs) & $M=17.5$ & $M=12.8$ & $M=24.8$ \\
& Min $=10$ & Min $=10$ & Min $=20$ \\
& Max $=33$ & Max $=17$ & Max $=33$ \\
& $S D=6.6$ & $S D=1.8$ & $S D=4.2$ \\
\hline Grade Levels & $\mathrm{n} / \mathrm{a}$ & $5^{\text {th }}$ to $11^{\text {th }}$ & $\mathrm{n} / \mathrm{a}$ \\
\hline Handedness & $85 \%$ right & $88 \%$ right & $81 \%$ right \\
\hline Expertise (self-report) & $0 \%$ beginner & $0 \%$ beginner & $0 \%$ beginner \\
& $39 \%$ average & $44 \%$ average & $31 \%$ average \\
& $59 \%$ expert & $52 \%$ expert & $69 \%$ expert \\
\hline
\end{tabular}

Table 1. Demographic information for the 41 participants. 


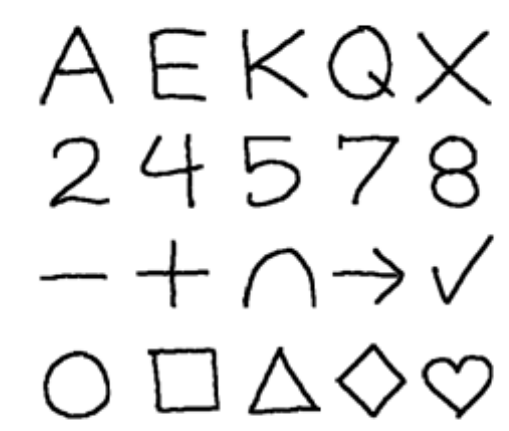

Figure 2. The set of $\mathbf{2 0}$ gesture types used in our study, which we borrow from prior work on gesture interaction for kids (Anthony et al., 2012).

crucial for gesture interaction design for children than for adults since children are still developing the sensorimotor coordination ability required to draw without looking.

\section{EXPERIMENT METHOD}

We conducted an empirical study with children, teens, and adults using mobile devices in a laboratory setting (Anthony, Brown, et al., 2013). Further work in this area will investigate more natural interaction outside of the laboratory, but for these initial explorations into the effect of visual feedback on interaction, collecting robust input data of specific types is necessary. We describe here the tasks performed by the participants and how visual feedback varied.

\subsection{Participants}

A total of 41 participants ( 25 children and teens, and 16 adults) participated in this study ( 20 were female). A demographic breakdown of the participants is given in Table 1. In general, the children in this study were preteens and teens. In future work, we plan to investigate younger children as well. Furthermore, most of the participants in our sample had experience using touchscreen devices such as smartphones and tablets and rated themselves either "average" or "expert" on a demographic questionnaire. In future work, we plan to investigate less expert users.

\subsection{Equipment}

We used Samsung Google Nexus S smartphones running the Android 4.0.4 operating system to conduct the experiment. The phones' dimensions were $4.88^{\prime \prime} \times 2.48^{\prime \prime} \times 0.43^{\prime \prime}$, and had 4 " screens, measured diagonally. Display resolution was $480 \times 800$ pixels (233 pixels per inch (ppi) pixel density). We created our own apps for this study that enabled us to log all input events generated by the participants during the study session.

\subsection{Procedure}

Participants came to a research laboratory to participate in the study. Up to three people could participate at one time (children/teens or adults only, no mixed sessions were conducted). Sessions lasted about one hour. During these sessions, participants engaged in a variety of tasks on mobile touchscreen devices. For the purposes of this paper, we present only one of these: the Gesture Task, which included both a Feedback and a No-Feedback condition, indicating the presence or lack of visual feedback. Participants were compensated \$10. 


\subsection{Gesture Input Task}

During the study, the participants drew gestures onscreen with their finger one at a time. There were 20 gestures used in the study, created based on existing mobile device apps as well as educational psychology literature about developmentally-appropriate gestures for children (Beery, Buktenica, \& Beery, 2004). (A similar task and gesture set has been used in prior work on gesture interaction for children (Anthony et al., 2012).) The gesture set (Figure 2 ) included letters, numbers, symbols, and geometric shapes ${ }^{6}$. Participants saw a prompt onscreen as to which gesture to enter (Figure 3a). To test the impact of visual feedback on gesture input, we included both a Feedback and a No-Feedback condition. In the Feedback condition, a trace was shown as the participant gestured (Figure 3b), but in the No-Feedback condition, no trace was shown. After entering the gesture, the participant touched the onscreen "Done" button to move on to the next gesture.

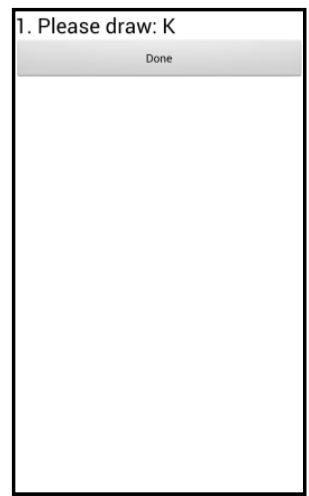

(a)

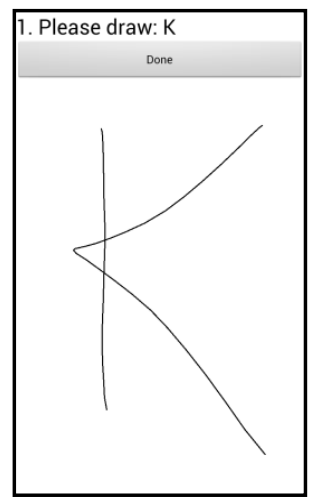

(b)

Figure 3. Screenshot of the study app's interface: (a) before drawing the gesture, and (b) after drawing the gesture, Feedback condition. (In NoFeedback, after drawing the gesture, the screen looked the same as (a).)

During the study, participants sat at a table in the lab and were allowed to hold the phone in a manner comfortable to them (e.g., handheld, resting on the table, etc.). Before doing the gesture input task on the phone, participants drew one sample of each gesture by hand on a sheet of paper. This activity helped ensure all of the gestures were familiar to the participants by name, since the app's prompt was textual (Figure 3). The app prompted the participant to enter one example of each gesture in the set one at a time, and then repeated this five times, yielding a total of 120 gesture samples ( 240 across both conditions per participant). Order of presentation of Feedback and No-Feedback tasks was counterbalanced across sessions (all participants in any one session completed them in the same order).

\subsection{Measures}

As participants dragged their finger across the device screen to enter each gesture, touch events were registered by the hardware and recorded by our app software for later data analysis. These touch events include

\footnotetext{
${ }^{6}$ Command gestures such as swipe and pinch-to-zoom were not included for two reasons: (1) studies find these gestures are difficult for children (Brown et al., 2011), and (2) many current children's educational apps use tracing or handwriting activities (Anthony et al., 2012).
} 
information such as the $x$-coordinate, $y$-coordinate, timestamp, touch pressure, and touch size of each event. A gesture might consist of multiple strokes; one stroke consists of all touch events registered between the time a finger-down and a finger-up event are registered. We used these data to calculate geometric properties of the gestures that were generated by each user, as well as to feed the stroke data into gesture recognition software to analyze recognition accuracy.

\section{ANALYSIS AND RESULTS}

In our study, we collected 9840 gestures across 41 participants. The first round of gestures in each condition was considered practice, and therefore is not included in our analysis, leaving a total of 8200 gestures. In our work, we typically consider 5 sub-groups of children/teens, based on age: 0 to 4 years, 5 to 7 years, 8 to 10 years, 11 to 13 years, and 14 to 17 years. These groups are derived from the following sources:

- developmental psychology literature (e.g., Piaget (Piaget, 1983));

- typical school age groupings in the United States (e.g., elementary school (5 to 10 years), middle school (11 to 13 years), and high school (14 to 17 years)); and,

- our experience conducting research with children and teens (Anthony, Brown, et al., 2013; Anthony et al., n.d., 2012; Brown \& Anthony, 2012).

In this study, children as young as 10 years of age participated, so our analyses are based on the following groups: 10 years ( 2 children), 11 to 13 years (16 children), 14 to 17 years ( 7 children), and adults (18+ years, 16 adults).

\subsection{Gesture Features}

Table 2 shows a list of the sixteen gesture features we analyzed in this paper, divided into two groups: (1) Simple features: (a) Number of (No.) strokes, (b) Number of (No.) points, (c) Gesture length, (d) Gesture height, (e) Gesture width, (f) Gesture area, (g) Gesture duration, (h) Gesture pressure, and (i) Gesture speed, and (2) Complex Features: (j) Gesture start angle, (k) Gesture end angle, (I) Gesture line similarity, (m) Gesture global orientation, ( $\mathrm{n}$ ) Gesture total turning angle, (o) Gesture sharpness, and ( $\mathrm{p}$ ) Gesture curviness; as well as how they were computed. Both types of features are geometric features that may be expected to impact recognition accuracy by making the gestures "look" different to the recognizer. While this list is by no means exhaustive, we believe it covers the most commonly used features and those most likely to affect interpretation of gesture input. Our original analysis included only the first 9 simple features (Anthony, Brown, et al., 2013). The Simple Features have been used in our prior work on gesture interaction, especially for children (Brown \& Anthony, 2012). The Complex Features have been recently utilized to uncover patterns in how adults make gestures (Anthony, Vatavu, et al., 2013); we here extend the use of these features to examine children's gestures. 


\begin{tabular}{|c|c|c|c|}
\hline & $\begin{array}{l}\text { Gesture } \\
\text { Feature }\end{array}$ & How Computed & Interpretation \\
\hline \multirow{9}{*}{ 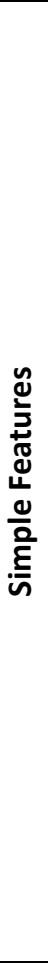 } & No. strokes (S) & $\begin{array}{l}\text { Total number of finger-down to finger-up periods } \\
\text { registered during a gesture. }\end{array}$ & $\begin{array}{l}\text { Number of times finger is lifted during one gesture, e.g., } \\
\text { Arch }->1 \text { stroke, } X->2 \text { strokes. }\end{array}$ \\
\hline & No. points (N) & $\begin{array}{l}\text { Total number of touch events registered during a } \\
\text { gesture, cumulatively over all strokes. }\end{array}$ & $\begin{array}{l}\text { Related to sampling speed of device: faster drawing } \\
\text { leads to fewer points registered, e.g., user, length, or } \\
\text { speed dependent. }\end{array}$ \\
\hline & Gesture length & $\begin{array}{l}\text { Cumulative path distance from the first touch event } \\
\text { registered for the gesture to the last. }\end{array}$ & How long the gesture path is, e.g., Circle $<Q$ path length. \\
\hline & Gesture height & $\begin{array}{l}\text { Height of the smallest bounding box that contains the } \\
\text { gesture }\left(\max _{y}-\min _{y}\right) \text {. }\end{array}$ & How tall the gesture is, e.g., Line $<$ Square height. \\
\hline & Gesture width & $\begin{array}{l}\text { Width of the smallest bounding box that contains the } \\
\text { gesture }\left(\max _{x}-\min _{x}\right) \text {. }\end{array}$ & How wide the gesture is, e.g., $8<$ Triangle width. \\
\hline & Gesture area & Gesture height * Gesture width. & How much area the gesture dimensions cover. \\
\hline & $\begin{array}{l}\text { Gesture } \\
\text { duration }\end{array}$ & $\begin{array}{l}\text { Time elapsed while drawing the gesture, e.g., time of } \\
\text { the last touch event registered for the gesture minus } \\
\text { time of the first touch event, including breaks } \\
\text { between strokes (milliseconds, or ms). }\end{array}$ & $\begin{array}{l}\text { How long it took to draw the gesture: faster gesture } \\
\text { entry or shorter gesture paths lead to lower durations. }\end{array}$ \\
\hline & $\begin{array}{l}\text { Gesture } \\
\text { pressure }\end{array}$ & $\begin{array}{l}\text { Average pressure registered over all the touch events } \\
\text { belonging to a gesture (pressure / N). }\end{array}$ & $\begin{array}{l}\text { How hard the user pressed onscreen while making the } \\
\text { gesture. }\end{array}$ \\
\hline & Gesture speed & $\begin{array}{l}\text { Average speed registered over all the touch events } \\
\text { belonging to a gesture (Gesture duration / Gesture } \\
\text { length). }\end{array}$ & $\begin{array}{l}\text { How quickly the user made the gesture, controlling for } \\
\text { length. }\end{array}$ \\
\hline \multirow{7}{*}{ 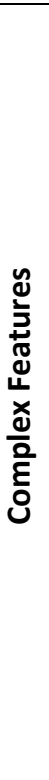 } & $\begin{array}{l}\text { Gesture start } \\
\text { angle }\end{array}$ & $\begin{array}{l}\text { Cosine of the initial angle of the gesture (Rubine } f_{1} \\
\text { feature (Rubine, 1991)). }\end{array}$ & $\begin{array}{l}\text { In what direction the user begins the gesture, e.g., } 5 \\
\text { tends to start straight left, } K \text { tends to start straight down. }\end{array}$ \\
\hline & $\begin{array}{l}\text { Gesture end } \\
\text { angle }\end{array}$ & Cosine of the ending angle of the gesture (Rubine $f_{1}$ ). & $\begin{array}{l}\text { From what direction the user ends the gesture, e.g., } A \\
\text { tends to end straight right, } 4 \text { tends to end straight down. }\end{array}$ \\
\hline & $\begin{array}{l}\text { Gesture line } \\
\text { similarity }\end{array}$ & $\begin{array}{l}\text { Distance between the starting and ending points / } \\
\text { Gesture length. }\end{array}$ & $\begin{array}{l}\text { How complex is the gesture path taken from start to end } \\
\text { point, controlling for length, e.g., Heart }<7 \text { line similarity }\end{array}$ \\
\hline & $\begin{array}{l}\text { Gesture global } \\
\text { orientation }\end{array}$ & $\begin{array}{l}\text { Angle of the diagonal of the gesture bounding box } \\
\text { (degrees) (Rubine } f_{4} \text { ). }\end{array}$ & $\begin{array}{l}\text { How skewed is the gesture compared to a vertical } \\
\text { orientation, e.g., Arrowhead }(\rightarrow)<K \text { global orientation } \\
\text { (short and wide vs. tall and narrow). }\end{array}$ \\
\hline & $\begin{array}{l}\text { Gesture total } \\
\text { turning angle }\end{array}$ & $\begin{array}{l}\text { Sum of the absolute value of the angles at each point } \\
\text { in the gesture (degrees) (Rubine } f_{10} \text { ). }\end{array}$ & $\begin{array}{l}\text { If a Circle is } 360^{\circ} \text {, how much total turning does the } \\
\text { gesture path demonstrate, e.g., Plus }<2 \text { total turning } \\
\text { angle (fewer curves in Plus). }\end{array}$ \\
\hline & $\begin{array}{l}\text { Gesture } \\
\text { sharpness }\end{array}$ & $\begin{array}{l}\text { Sum of the squared angles at each gesture point } \\
\text { (degrees) (Rubine } f_{11} \text { ). }\end{array}$ & $\begin{array}{l}\text { How sharp are the corners and direction changes as the } \\
\text { user's gesture progresses, e.g., Circle < Square sharpness } \\
\text { (same shape but square has sharp corners). }\end{array}$ \\
\hline & $\begin{array}{l}\text { Gesture } \\
\text { curviness }\end{array}$ & $\begin{array}{l}\text { Total turning angle / Gesture length (degrees / pixel) } \\
\text { (Long et al (Long, Landay, Rowe, \& Michiels, 2000), } \\
\text { feature 13). }\end{array}$ & $\begin{array}{l}\text { Degree of curvature in the user's gesture strokes, } \\
\text { controlling for length, e.g. } E<\text { Heart curviness (fewer } \\
\text { curves in } E \text {, normalized by length). }\end{array}$ \\
\hline
\end{tabular}

Table 2. Gesture features analyzed in this paper. 


\begin{tabular}{|c|c|c|c|c|}
\hline & & \multicolumn{3}{|c|}{ Significant Results } \\
\hline & Gesture Feature & $\begin{array}{l}\text { Participant } \\
\text { Age Group } \\
\text { Main Effect }\end{array}$ & $\begin{array}{c}\text { Visual } \\
\text { Feedback? } \\
\text { Main Effect }\end{array}$ & $\begin{array}{c}\text { Participant Age Group } \\
\text { x Visual Feedback? } \\
\text { Interaction }\end{array}$ \\
\hline \multirow{9}{*}{ 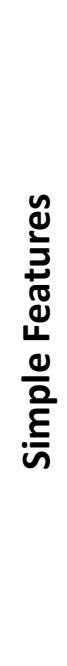 } & No. strokes & - & - & - \\
\hline & No. points & - & - & ++ \\
\hline & Gesture length & - & ++ & ++ \\
\hline & Gesture height & - & ++ & ++ \\
\hline & Gesture width & - & ++ & ++ \\
\hline & Gesture area & - & ++ & ++ \\
\hline & Gesture duration & - & ++ & ++ \\
\hline & Gesture pressure & - & ++ & ++ \\
\hline & Gesture speed & - & ++ & ++ \\
\hline \multirow{7}{*}{ 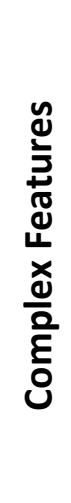 } & Gesture start angle & - & ++ & - \\
\hline & Gesture end angle & - & + & + \\
\hline & Gesture line similarity & - & ++ & - \\
\hline & Gesture global orientation & - & ++ & ++ \\
\hline & Gesture total turning angle & - & + & ++ \\
\hline & Gesture sharpness & - & + & ++ \\
\hline & Gesture curviness & + & ++ & ++ \\
\hline
\end{tabular}

Table 3. Significant interactions and main effects for each gesture feature of participant age group and visual feedback?. + indicates $p<.05$ and ++ denotes $p<.01,-$ indicates $p>.05$.

\subsubsection{Simple Features}

We analyzed each feature for gestures created by children and adults in the presence and absence of visual feedback. For the Simple Features, we conducted a series of ANOVA tests to determine where differences may lie for each feature. In all cases, we conducted a univariate ANOVA with participant age group and visual feedback? as fixed factors. Because each participant entered multiple gestures, we included participant as a random factor ${ }^{7}$. Because the study design was nested (e.g., participants could only be in one age group), we

${ }^{7}$ A random factor's levels have been chosen at random and might change when doing the study again (e.g., participants drawn from the population). It is an accepted practice to use participant as a random factor for repeated measures when the number of samples per participant is very many or not equal (Dean \& Voss, 1999) (p. 630). 
constructed a model with the main effect terms for participant age group and visual feedback?, a nested term for participant(participant age group), and an interaction term for participant age group $\mathrm{x}$ visual feedback?. Table 3(a) summarizes the significant effects; specific findings for each feature are discussed below.

No. strokes. The number of strokes showed no significant differences based on participant age group $\left(F_{3,37}=\right.$ 1.00 , n.s. $)$, visual feedback? $\left(F_{1,8154}=3.72\right.$, n.s. $)$, or their interaction $\left(F_{3,8154}=0.42\right.$, n.s. $)$. Because the interaction was not significant, we re-ran the ANOVA without the interaction in the model; this time there was a significant main effect of visual feedback? $\left(F_{1,8157}=9.01, p<.01\right)$. Thus, although the number of strokes was not a distinctive feature between age groups, it was responsive to the presence or absence of visual feedback. Users tended to generate gestures with fewer strokes with no visual feedback.

No. points. The number of points sampled during a gesture showed a significant interaction between participant age group and visual feedback? $\left(\mathrm{F}_{3,8154}=15.47, p<.01\right)$. We see a shift from the younger children to the adults, in which 10 year olds and 11 to 13 year olds tend to make gestures more quickly in the absence of visual feedback, but 14 to 17 year olds show no difference, and adults tend to make them more quickly in the presence of visual feedback.

Gesture length. There was a significant interaction between participant age group and visual feedback? $\left(\mathrm{F}_{3,8154}=\right.$ $4.03, p<.01$ ). All age groups tended to make shorter (length) gestures in the presence of visual feedback, but the youngest children and adults showed a smaller difference than the middle age groups.

Gesture height. There was a significant interaction between participant age group and visual feedback? $\left(\mathrm{F}_{3,8154}=\right.$ $15.14, p<.01$ ), in which most age groups made shorter (height) gestures in the presence of visual feedback, except the 10-year-olds.

Gesture width. The width of the gestures generated showed a significant interaction between participant age group and visual feedback? $\left(\mathrm{F}_{3,8154}=14.10, p<.01\right)$. Most age groups made narrower gestures in the presence of visual feedback, but what varied was the degree of difference (smaller for adults).

Gesture area. As gesture area is a composite of gesture height and gesture width, it is perhaps unsurprising that this feature showed the same relationship again: a significant interaction was found between participant age group and visual feedback? $\left(\mathrm{F}_{3,8154}=25.84, p<.01\right)$.

Gesture duration. The amount of time taken to draw a gesture showed a significant interaction between participant age group and visual feedback? $\left(\mathrm{F}_{3,8154}=5.80, p<.01\right)$. The younger children (10 year olds and 11 to 13 year olds) tended to take more time to draw gestures in the presence of visual feedback vs. absence of visual feedback than did 14 to 17 year olds and adults.

Gesture pressure. The average pressure exerted by the participant's finger during a gesture also showed a significant interaction between participant age group and visual feedback? $\left(\mathrm{F}_{3,8154}=31.75, p<.01\right)$. All age groups exerted less pressure in the presence of visual feedback, but this difference was more pronounced for the 10 -year-olds.

Gesture speed. The average speed of a gesture is related to the length and the duration, so unsurprisingly, this feature showed the same relationship: a significant interaction was found between participant age group and 
visual feedback? $\left(\mathrm{F}_{3,8154}=26.03, p<.01\right)$. All age groups tended to draw gestures faster in the absence of visual feedback, but for adults, this effect was less pronounced.

\subsubsection{Complex Features}

For the Complex Features, we also conducted a series of ANOVA tests to determine where differences may lie for each feature. For these features, there is likely to be a greater impact of gesture type (e.g., any 5 gesture and $K$ gesture will have very different gesture start angles just based on global execution patterns), so we controlled for the type of the gesture being made in these tests. In all cases, we conducted a univariate ANOVA with participant age group, visual feedback?, and gesture type as fixed factors. Again, because each participant entered multiple gestures, we included participant as a random factor. Because the study design was nested (e.g., participants could only be in one age group), we constructed a model with the main effect terms for participant age group, visual feedback?, and gesture type; a nested term for participant(participant age group); and an interaction term for participant age group $\mathrm{x}$ visual feedback?. Table 3(b) summarizes the significant effects; specific findings for each feature are discussed below.

Gesture start angle. There was no significant interaction between participant age group and visual feedback? $\left(F_{3,8128}=1.94\right.$, n.s. $)$, nor was there a main effect of participant age group $\left(F_{3,37}=1.61\right.$, n.s. $)$. However, there was a significant main effect found for visual feedback? $\left(\mathrm{F}_{1,8128}=6.96, p<.01\right)$. Generally, users tended to start their gestures with a slightly steeper start angle in the absence of visual feedback, although the effect is very small.

Gesture end angle. The angle at which the user ended the gesture showed a significant interaction between participant age group and visual feedback? $\left(\mathrm{F}_{3,7278}=3.43, p<.05\right)$. For all age groups except the youngest (10year-olds), users tended to end their gestures with a slightly steeper closing stroke in the presence of visual feedback. The youngest age group exhibited the opposite behavior.

Gesture line similarity. The gesture's similarity to a line (e.g., reflecting a measure of the complexity of the gesture) showed no significant interaction between participant age group and visual feedback? $\left(\mathrm{F}_{3,8134}=0.25\right.$, n.s.), nor was there a significant main effect of participant age group $\left(\mathrm{F}_{3,37}=0.14\right.$, n.s.). However, there was a significant main effect for visual feedback? $\left(\mathrm{F}_{1,8134}=15.84, p<.01\right)$. For all age groups, users tended to make gestures that were more similar to lines in the presence of visual feedback; that is, they tended to draw straighter lines with more efficiency and less wiggling or wobbling.

Gesture global orientation. There was a significant interaction between participant age group and visual feedback? $\left(\mathrm{F}_{3,8134}=5.34, p<.01\right)$. We see a shift from the younger children to the adults, in which the children all tended to make gestures with a slightly less steep global orientation (e.g., skewed to the right or to the left) in the absence of visual feedback, but to a lesser degree for the older children than for the younger children. For adults, there was no difference between the feedback conditions in the global orientation, controlling for gesture type.

Gesture total turning angle. The cumulative angle through which the gesture was drawn showed a significant interaction between participant age group and visual feedback? $\left(F_{3,8134}=14.83, p<.01\right)$. We again see a shift from the younger children to the adults, in which 10 year olds and 11 to 13 year olds tended to make gestures with a larger turning angle in the presence of visual feedback, whereas for 14 to 17 year olds we see no difference, and adults exhibit the opposite behavior. 
Gesture sharpness. There was a significant interaction between participant age group and visual feedback? $\left(F_{3,8134}=9.71, p<.01\right)$. Again, we see a shift in behavior from the youngest to oldest participants in our study: 10 year olds and 11 to 13 year olds tended to make gestures with a higher degree of sharpness in the presence of visual feedback, whereas 14 to 17 year olds showed no difference, and adults exhibited the opposite behavior. We also saw a marginal trend in which adults tended to make overall sharper gestures than children of any age $\left(\mathrm{F}_{3,37}=2.56, p<.07\right)$.

Gesture curviness. There was a significant interaction between participant age group and visual feedback? $\left(F_{3,8134}=33.26, p<.01\right)$. This feature also exhibited a similar shift for participants of different age groups: adults and 14 to 17 year olds tended to make gestures of roughly the same curviness in both visual feedback conditions, whereas 10 year olds and 11 to 13 year olds tended to make "curvier" gestures in the presence of visual feedback. Also, adults tended to make overall curvier gestures than children of any age $\left(F_{3,37}=3.94, p<\right.$ $.05)$.

\subsubsection{Features Discussion}

It is worthy of note that for all features we examined (both Simple and Complex), there was a significant effect of the nested term participant(participant age group), indicating a strong influence of individual differences on gesture behavior. This occurrence is consistent with prior work in handwriting recognition (Crettez, 1995; Srihari, Cha, Arora, \& Lee, 2001) and multitouch gestures (Schmidt \& Weber, 2010). Because this factor was accounted for in the model, our statistical results showing the effects of the other factors are reliable. We simply note this factor and consider the possibility of examining individual behavior under different feedback conditions in more detail as future work. Also, all Complex Gestures did show a significant effect of gesture type; as we predicted, it was necessary to control for this factor when analyzing the feature differences by age group and visual feedback?

The Simple Features show a general trend that users are more careful when generating gestures in the presence of visual feedback (shown by gesture duration and gesture speed). This behavior could be due to an increased fluency in entering gestures when users can see what they are doing; the visual feedback provides a "check" on the sensorimotor feedback they get while drawing a gesture, increasing confidence. The generation of gestures with fewer strokes in the absence of visual feedback was unexpected, but when one considers the challenge of joining strokes without visual feedback once a finger has been lifted, it becomes more clear. Finally, the shorter and more compact gestures that were made in the presence of visual feedback could also be due to a fluency effect: being able to see one's trace visually can increase confidence in finer-grained movements.

The Complex Features show a more involved picture. Four of the features point to the complexity of the path the user follows as he or she makes the gesture (gesture line similarity, gesture total turning angle, gesture sharpness, and gesture curviness). In general, we saw an increase in complexity, reflected in meandering paths and less crisp execution, when there was no visual feedback, supporting our findings for the Simple Features that there is a lack of fluency imposed by removing visual feedback. We also see that presence or absence of visual feedback has an impact on gesture direction and orientation (gesture start angle, gesture end angle, and gesture global orientation). For example, global orientation was skewed tall and thin in the presence of visual feedback vs. short and squat in the absence of it. We speculate that this indicates a higher degree of accommodation to the aspect ratio of the device; the smartphone screen was taller than it was wide, and users 
tend to be more cautious to not go too near the screen edges when they could see their gesture than when they could not.

In most cases, for both the Simple and the Complex Features, the younger the children, the greater degree of variation they exhibited between gestures they created in the presence or absence of visual feedback. This effect is a strong indicator that children struggle without visual feedback. However, even adults show some variation between the two cases, and as a result, we recommend that visual feedback always be provided during surface gesture interaction with these types of input gestures. Users of all ages can benefit from this accommodation.

\subsection{Gesture Recognition}

Differences in how children and adults make gestures in the presence or absence of visual feedback may not actually be relevant to the design of gesture interaction on mobile devices if it is equivalently easy (or difficult) to recognize these gestures. To understand the impact on recognition of the significant differences we discovered in these gesture features, we ran the gestures through the $\$ N$-Protractor recognizer (Anthony \& Wobbrock, 2012) and the \$P recognizer (Vatavu, Anthony, \& Wobbrock, 2012). Both of these recognizers are accurate, trainable, and open-source, and are widely used by gesture interaction researchers and mobile app developers. We conducted user-dependent training, in which we first trained each of the gesture recognizers on a small set of one user's gestures (evenly sampling from all gesture types) and then tested the recognizer on the remainder of that user's samples. We repeated this procedure for all users, computing average per-user recognition accuracy for each of the age groups. Note that we conducted separate tests for gestures generated in the presence of visual feedback and those generated in its absence, so that we could compare the effect of visual feedback per participant. We then aggregated these results across participants. Table 4 shows all recognition results by age group separated by presence or absence of visual feedback for both recognizers.

For each of the two different recognizers, we conducted a separate repeated-measures ANOVA on the per-user recognition accuracy on the within-subjects factor of visual feedback? and the between-subjects factor of

\begin{tabular}{|r|l|c|c|c|c|c|c|}
\hline & & \multicolumn{3}{|c|}{ \$N-Protractor } & \multicolumn{3}{c|}{ \$P } \\
\hline $\begin{array}{r}\text { Age } \\
\text { Group }\end{array}$ & Condition & Mean & SD & N & Mean & SD & N \\
\hline \multirow{2}{*}{$\mathbf{1 0}$ yrs } & Feedback & $77.1 \%$ & $7.8 \%$ & 2 & $88.8 \%$ & $0.5 \%$ & 2 \\
\cline { 2 - 8 } & No Feedback & $77.6 \%$ & $6.4 \%$ & 2 & $80.8 \%$ & $8.6 \%$ & 2 \\
\hline \multirow{2}{*}{$\mathbf{1 1}$ to $\mathbf{1 3}$} & Feedback & $80.7 \%$ & $7.8 \%$ & 16 & $91.4 \%$ & $6.8 \%$ & 16 \\
\cline { 2 - 8 } & No Feedback & $84.2 \%$ & $5.4 \%$ & 16 & $90.3 \%$ & $5.5 \%$ & 16 \\
\hline \multirow{2}{*}{$\mathbf{1 4}$ to $\mathbf{1 7}$} & Feedback & $87.6 \%$ & $5.4 \%$ & 7 & $95.9 \%$ & $2.5 \%$ & 7 \\
\cline { 2 - 8 } & No Feedback & $87.2 \%$ & $7.8 \%$ & 7 & $95.3 \%$ & $4.8 \%$ & 7 \\
\hline $\begin{array}{r}\text { Adults } \\
(\mathbf{1 8 + )}\end{array}$ & Feedback & $90.8 \%$ & $6.4 \%$ & 16 & $97.0 \%$ & $2.9 \%$ & 16 \\
\cline { 2 - 8 } & No Feedback & $90.8 \%$ & $4.9 \%$ & 16 & $96.9 \%$ & $3.3 \%$ & 16 \\
\hline
\end{tabular}

Table 4. Recognition accuracy of \$N-Protractor (Anthony \& Wobbrock, 2012) and \$P (Vatavu et al., 2012) on gestures by age group and presence or absences of visual feedback. 
participant age group. For \$N-Protractor, reported in our original analysis (Anthony, Brown, et al., 2013), we found no significant difference in accuracy based on visual feedback? $\left(F_{1,37}=0.56\right.$, n.s. $)$, nor was the interaction with participant age group significant $\left(\mathrm{F}_{3,37}=1.45\right.$, n.s. $)$, but we did find a significant main effect of participant age group alone $\left(\mathrm{F}_{3,37}=7.38, p<.01\right)$. For $\$ \mathrm{P}$, generally recognition accuracy was higher than with $\$ \mathrm{~N}$-Protractor, supporting prior work (Anthony et al., n.d.). Although the interaction between visual feedback? and participant age group was not significant for $\$ P\left(\mathrm{~F}_{3,37}=2.07\right.$, n.s. $)$, we did find a significant main effect of visual feedback? $\left(\mathrm{F}_{1,37}=6.91, p<.05\right)$, as well as a significant main effect of participant age group $\left(\mathrm{F}_{3,37}=8.79, p<.01\right)$.

Supporting prior work on recognition of children's gestures with current recognizers (Anthony et al., n.d., 2012; Brown \& Anthony, 2012), we find that recognizing children's gestures is still harder than recognizing adults' gestures, for both recognizers. The younger the children are, the lower recognition accuracy is. However, we see an interesting divergence in the two recognizers in terms of whether their accuracy is affected by the presence or absence of visual feedback: \$N-Protractor shows no difference, while \$P does have more difficulty recognizing gestures made in the absence of visual feedback. When we additionally consider the differences already noted in the features that make up these gestures, this finding implies that there is a stronger correlation between the features that differed and the features that the \$P recognizer uses to classify gestures than those that $\$ \mathrm{~N}-$ Protractor uses.

While neither of the recognizers explicitly uses any of the sixteen features in its recognition process, changes in these features clearly lead to different behaviors for \$P. For example, \$P simplifies all gestures it recognizes by resampling (equalizing the number of points), resizing (equalizing the height, width, and area), and considering the points individually rather than grouped by strokes (equalizing number of strokes). The features most likely to impact \$P's performance are those that increase the geometric complexity of the layout of the individual points of the gesture. Both line similarity and total turning angle are candidate features that could affect this level of detail. To characterize this space, we computed bivariate correlations between \$P's recognition accuracy per gesture type and the average value of the different features by gesture type, separated into presence and absence of visual feedback. Table 5 shows the significant correlations. Four features show significant correlations with the recognition rate: number of strokes, gesture length, gesture duration, and gesture line similarity. From the results in the previous section, all of these features were significantly impacted by the presence of absence of visual feedback.

\begin{tabular}{|l|c|c|c|c|c|c|c|c|}
\hline & $\begin{array}{c}\text { No. } \\
\text { strokes }\end{array}$ & $\begin{array}{c}\text { No. } \\
\text { points }\end{array}$ & $\begin{array}{c}\text { Gesture } \\
\text { length }\end{array}$ & $\begin{array}{c}\text { Gesture } \\
\text { height }\end{array}$ & $\begin{array}{c}\text { Gesture } \\
\text { width }\end{array}$ & $\begin{array}{c}\text { Gesture } \\
\text { area }\end{array}$ & $\begin{array}{c}\text { Gesture } \\
\text { duration }\end{array}$ & $\begin{array}{c}\text { Gesture } \\
\text { pressure }\end{array}$ \\
\cline { 2 - 9 } Correlation to \$P & $-.40^{*}$ & - & $-.56^{* *}$ & - & - & - & $-.37^{*}$ & - \\
\cline { 2 - 9 } Recognition Rate & - & - & - & $.43^{* *}$ & - & - & - & - \\
\cline { 2 - 8 } & $\begin{array}{c}\text { Gesture } \\
\text { speed }\end{array}$ & $\begin{array}{c}\text { Gesture } \\
\text { start } \\
\text { angle }\end{array}$ & $\begin{array}{c}\text { Gesture } \\
\text { end } \\
\text { angle }\end{array}$ & $\begin{array}{c}\text { Gesture } \\
\text { line } \\
\text { similarity }\end{array}$ & $\begin{array}{c}\text { Gesture } \\
\text { global } \\
\text { orientation }\end{array}$ & $\begin{array}{c}\text { Gesture } \\
\text { total } \\
\text { turning } \\
\text { angle }\end{array}$ & $\begin{array}{c}\text { Gesture } \\
\text { sharp- } \\
\text { ness }\end{array}$ & $\begin{array}{c}\text { Gesture } \\
\text { curvi- } \\
\text { ness }\end{array}$ \\
\hline
\end{tabular}

Table 5. Correlations between \$P's recognition rate and the geometric features we have analyzed in this paper, per gesture type. * denotes significance at the $p<.05$ level, ** denotes the $p<.01$ level. 


\begin{tabular}{|l|c|c|c|c|}
\hline & \multicolumn{2}{|c|}{ Adults } & \multicolumn{2}{c|}{ Children } \\
\cline { 2 - 5 } & \$N-Protractor & \$P & \$N-Protractor & \$P \\
\hline \multirow{3}{*}{ Presence of } & Triangle & $\mathrm{A}$ & Diamond & Triangle \\
Visual & $\mathrm{A}$ & $\mathrm{E}$ & Triangle & $\mathrm{E}$ \\
Feedback & $\mathrm{E}$ & $\mathrm{X}$ & Arrowhead & $\mathrm{K}$ \\
& Diamond & Line & Rectangle & $\mathrm{A}$ \\
& Circle & Triangle & Heart & $\mathrm{X}$ \\
\hline \multirow{3}{*}{ Absence of } & Triangle & $\mathrm{X}$ & Rectangle & Rectangle \\
Visual & Rectangle & Rectangle & Triangle & $\mathrm{A}$ \\
Feedback & Arrowhead & $\mathrm{E}$ & A & $\mathrm{E}$ \\
& Diamond & $\mathrm{A}$ & Plus & Triangle \\
& $\mathrm{A}$ & Diamond & Diamond & $\mathrm{X}$ \\
\hline
\end{tabular}

Table 6. Top five worst recognized gestures for children and adults, with and without visual feedback, for both recognizers used in this paper.

This analysis provides a deeper understanding of the exact mechanisms by which the ways users make gestures are related to the recognition of those gestures, depending on the presence or absence of visual feedback. What we can conclude is that, for some recognizers, there do exist critical features which vary in the presence or absence of visual feedback in such a way as to (negatively) affect recognition. Because \$P was significantly more accurate on children's and adults' gestures than was $\$ \mathrm{~N}$-Protractor, it is more desirable to use \$P in applications. However, \$P's accuracy was affected by whether the gestures were entered with the presence or absence of visual feedback; therefore, we consider these findings as evidence in favor of providing visual feedback when using recognizers such as $\$ \mathrm{P}$ to provide the best accuracy and user experience.

\subsection{Qualitative Observations}

We observed anecdotally that participants seemed confused by the absence of visual feedback while they were performing the gesture task. Participants commented that they could not see their finger markings to help them enter the gestures, and that they didn't like not being able to see what was being drawn. These comments were especially common if the participants had done the Feedback condition first. We noted that several of the 14 to 17 year old participants expressed pleasure when they could see their gestures and were happy with how they appeared. This effect diminished in later sessions when we warned participants that they would not see their gestures some of the time, but the gestures they created in the absence of visual feedback remained poorer from a qualitative perspective (as well as the quantitative feature differences already discussed). Figure 4 shows a few comparative examples of gestures drawn by children in the Feedback and No-Feedback conditions. These differences caused a decrease in recognition rates for \$P. They are also clearly visually different, demonstrating the differences we found in both the simple gesture features (e.g., gesture length, height, width, etc.), and the complex gesture features (e.g., line similarity, gesture curviness). Other issues are also apparent (e.g., redrawing the rectangle).

Although eventually all users completed all gestures in both the presence and absence of visual feedback, users' frustration in the latter case did not seem to diminish as the study session went on. Especially given the lower quality of gestures in the No-Feedback condition, we suggest that these observations indicate that gesture 
interaction without visual feedback does not feel comfortable to users, especially children, and is not recommended.

\subsection{Discussion}

In general, we have found evidence to mostly support the use of visual feedback during gesture interaction. In terms of geometric properties of the gestures drawn by our participants, all age groups (children, teens, and adults) made different gestures in the presence of visual feedback than they did in its absence. These differences did not impact the ability of one gesture recognizer ( $\$ N$-Protractor (Anthony \& Wobbrock, 2012)) to classify the gestures, but they did impact the ability of another (\$P (Vatavu et al., 2012)) to do so. Since \$P was more accurate than $\$ \mathrm{~N}$-Protractor on both children's and adults' gestures, it is preferable to use $\$ \mathrm{P}$ in applications. However, the challenges $\$ P$ had with gestures entered in the absence of visual feedback cause us to recommend in favor of providing visual feedback for gesture interaction for the best accuracy and user experience. In addition, when we consider users' opinions on using interfaces with and without visual feedback, we find further reason to recommend the inclusion of visual feedback.

We here reflect in more detail how to understand the reasons why the two recognizers, in spite of the differences in gesture features, showed different effects of presence or absence of visual feedback. We examine which gestures were the most challenging for the recognizers in our tests. Table 6 lists the five gestures that were the least accurately recognized by $\$ \mathrm{~N}$-Protractor and \$P when considering participant age group (just adults vs. children) and visual feedback?. The lists for children vs. adults and Feedback vs. No Feedback are very similar for both recognizers, leaving an inconclusive understanding as to how specific gestures that are made with inconsistent features between visual feedback conditions pose difficulty to $\$ P$ but not to $\$ N-P r o t r a c t o r$. Gestures such as triangle and $A$, which were the least well recognized by $\$ P$ in our tests, tend to be shorter (gesture length), are made less quickly (gesture duration), and tend to be tidier without extra wobbles (gesture line similarity) in the presence of visual feedback. We suggest that these gestures are either particularly difficult for participants to draw when they cannot see a visual trace of their path, or the gestures are particularly challenging in general for \$P. Anecdotally, we did observe during the study that gestures like the arrowhead and diamond were not as familiar to the participants, especially the children, as were the other types of gestures. Furthermore, \$P could be expected to have difficulty distinguishing between triangles and A's, which are very similar geometrically. More exploration of the types of errors made by the recognizer (e.g., which gestures are confused for each other most often) is needed to answer this question sufficiently. With such analysis, it could be possible to design gesture sets to ensure consistency by users, and to use only gestures that are wellrecognized by the system.

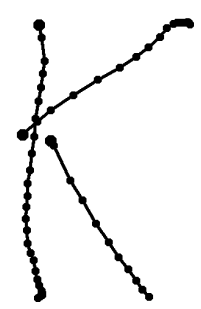

(a) with feedback

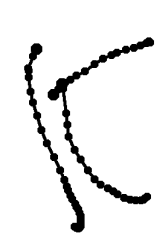

(b) without feedback
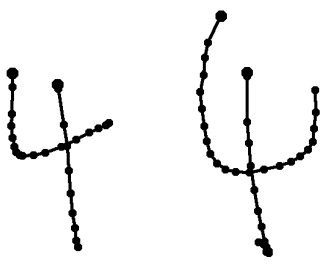

(c) with feedback (d) without feedback

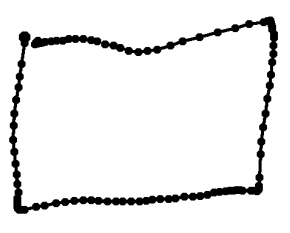

(e) with feedback

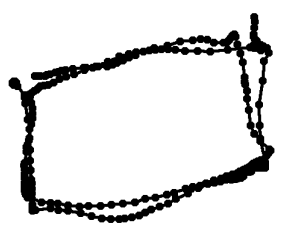

(f) without feedback

Figure 4. Examples of gestures produced with and without visual feedback 
Also, some prior work has examined differences between features of surface gestures generated by children and adults, in one case finding a difference (Brown \& Anthony, 2012), and in another case not finding one (Anthony et al., 2012). We believe the work we report in this paper can settle the discrepancy between these two prior studies. In the study by Anthony et al. (Anthony et al., 2012), in which no gesture feature differences were found between adults and children for a similar gesture input task, only a Feedback condition was tested. In the study by Brown et al. (Brown \& Anthony, 2012), in which differences were found (number of strokes, gesture height, gesture duration, and gesture pressure), they used a gesture input task with No Feedback. Neither of these studies tested both the presence and absence of visual feedback, as we have done here. When we consider both of these similar prior studies and the interactions we have found between participant age group and visual feedback? in this study, we can conclude that the primary factor contributing to gesture generation differences among children and adults is whether or not there is visual feedback provided. When visual feedback is used, participants are more comfortable and generate more consistent gestures. When it is not used, participants' input behaviors are less consistent, and this effect is magnified for children over adults. Therefore, we believe that the cumulative evidence across these three studies favors use of visual feedback for these types of gestures during surface gesture interaction.

\section{DESIGN IMPLICATIONS}

Based on the findings from the study presented in this paper, we outline four new design recommendations for surface gesture interaction on mobile devices for children, teens, and adults.

DO provide visual feedback for surface gesture interaction on mobile devices. We found evidence that users' gestures are made differently in the presence than in the absence of visual feedback. Although in this study it only impacted recognition results for one recognizer, users expressed dissatisfaction with surface gesture interactions without visual feedback. Allowing users to see the trace of their finger's path along the device screen can improve carefulness and confidence in their input. Although this recommendation can improve interaction for users of all ages, it is particularly relevant to interaction design for children. Children's mental agility in imagining their finger's path is less well-developed than that of adults, and therefore visual feedback can aid them in developing this hand-eye coordination skill as they mature.

DON'T include gestures unfamiliar to users. When designing gesture sets for new applications, it is risky to use new gestures that users may not already know how to draw. More commonly used shapes that users encounter outside of their interactions with a given application will be more comfortable for them, increasing the consistency with which they generate gestures. In turn, these gestures will be more easily recognized by the system. This consideration applies to users of all ages, but is especially critical for interaction design for children. Children have less experience with technology, less schooling and exposure to the range of possible letters and shapes (Beery et al., 2004), and less developed fine-motor control, which impacts the dexterity of this population. Designers of application gesture sets should consider both the requirements of the algorithms along with the cognitive abilities of their users.

DON'T include gesture pairs that are geometrically similar or likely to appear similar to recognizers. In our study, we saw that \$P tends to have difficulty recognizing gestures such as triangle and $A$, which are very similar to each other visually. In some cases, it may not be so obvious which gestures will appear similar to recognizers. For example, prior work has found that $\mathrm{A}$ and $\mathrm{K}$ tend to be confused by $\$ \mathrm{~N}$-Protractor because the pre- 
processing steps taken during recognition cause the two gestures to appear more similar (Anthony et al., 2012). In these cases, it is better to reduce the gesture set size by avoiding having both confusable gestures in the set: include one or the other, but not both. Understanding the limitations of the recognizer is even more important when supporting gesture interaction for children, who tend to have higher proportions of misrecognized gestures.

DO test new gesture sets with the target recognizer in advance. When designing gesture-based interaction, the recognition approach can make a difference in how well users' gestures are understood. We have tested two of the current state-of-the-art approaches, \$N-Protractor (Anthony \& Wobbrock, 2012) and \$P (Vatavu et al., 2012). Only $\$ P^{\prime}$ s recognition accuracy was sensitive to the presence of absence of visual feedback, even though it was more accurate overall. Furthermore, both recognizers made mistakes on very different gestures: $\$ \mathrm{~N}$ Protractor classified basic shapes more poorly (e.g., triangle, diamond, rectangle), and \$P classified letters more poorly (e.g., A, E, X). In order to identify the gestures that will be challenging, or pairs that will be confusing, early testing is critical. A key design recommendation for surface gesture interaction, especially with children, is to use iterative rapid prototyping that can expose conflicts (either from the user's or system's perspective) in the gesture set early.

\section{FUTURE WORK}

This work is the first to explore the impact of visual feedback on surface-gesture input for children, teens, and adults, and as such represents a foundational study in this space. Many other factors may also be relevant to successful gesture-based interaction design for children, and we briefly list a few that we have identified as promising areas of future work. First, we have included a wide age range of children in this study, from 10 to 17 years old (and adults from 20 to 33 years old). This work characterizes the impact of visual feedback on gesture generation for older children who are fairly comfortable with writing and drawing activities, and it may be informative to extend this work to younger children who are just starting out in school (ages 5 to 9) or even preschool-aged children (ages 1 to 4). We anticipate that the impact of visual feedback will be more pronounced for these younger children. We also think that validating these results with children, teens, and adults of varying levels of experience with mobile touchscreen devices and gesture interaction will be important to fully explore this space.

Second, we have examined a fairly abstracted task, in which the participants were entering samples of the gestures without a goal for using that gesture to do anything (e.g., to launch a task or respond to a query). We do not yet know how a change in the user's goal might interact with the user's input with or without visual feedback. In some handwriting practice activity apps for children that exist today (e.g., Jaloby's AlphaCount ${ }^{8}$ ), the interface may be only a little more embellished than our app to prompt the child for a gesture to draw. Thus, we believe that this abstracted task makes a good foundation, and plan to extend it to contextualized tasks in future work. We expect to see similar patterns, but predict a decrease in the impact of the absence of visual feedback for tasks where there is important information onscreen that the gesture might otherwise obscure.

\footnotetext{
${ }^{8}$ https://itunes.apple.com/us/app/alphacount/id359046783
} 


\section{CONCLUSION}

We have presented the results of an empirical study examining the impact of the presence or absence of visual feedback during surface gesture input on a mobile device. Our findings from data from 41 children, teens, and adults indicate that gestures generated with and without visual feedback differ significantly in ways that make them difficult to interpret by the current state-of-the-art recognizers that we tested. For example, users tend to make gestures with fewer strokes in the absence of visual feedback. They also tend to make shorter, more compact gestures using straighter lines with more efficiency and less wobbling in the presence of visual feedback. In addition, users of all age groups we studied prefer to see visual feedback, although adults are more willing to accept lack of feedback. Based on our findings, we present design recommendations for new surface gesture interfaces for children, teens, and adults regarding the use of visual feedback. The results of this work will be informative to designers and researchers interested in surface gesture interaction on mobile devices for all ages.

\section{ACKNOWLEDGMENTS}

This work was partially supported by Department of Education HBGI Grant Award \#P031B090207-11 and National Science Foundation Grant Awards \#IIS-1218395/IIS-1218664. Any opinions, findings, and conclusions or recommendations expressed in this paper are those of the authors and do not necessarily reflect these agencies' views. We would also like to thank our collaborators at Bowie State and UMBC, including Robin Brewer, Thaddeus Brown, Germaine Irwin, Shreya Mohan, Monique Ogburn, and Chiamaka Okorohoa for project support.

\section{REFERENCES}

Akamatsu, M., MacKenzie, I. S., \& Hasbroucq, T. (1995). A comparison of tactile, auditory, and visual feedback in a pointing task using a mouse-type device. Ergonomics, 38(4), 816-827. doi:10.1080/00140139508925152

Anthony, L., Brown, Q., Nias, J., \& Tate, B. (2013). Examining the Need for Visual Feedback during Gesture Interaction on Mobile Touchscreen Devices for Kids. Proceedings of the International Conference on Interaction Design and Children (p. to appear).

Anthony, L., Brown, Q., Nias, J., Tate, B., \& Mohan, S. (2012). Interaction and Recognition Challenges in Interpreting Children's Touch and Gesture Input on Mobile Devices. Proceedings of the ACM International Conference on Interactive Tabletops and Surfaces (pp. 225234). ACM Press.

Anthony, L., Brown, Q., Tate, B., Nias, J., Brewer, R., \& Irwin, G. (n.d.). Designing Smarter Touch-Based Interfaces for Educational Contexts. Journal of Personal and Ubiquitous Computing: Special Issue on Educational Interfaces, Software, and Technology, to appear.

Anthony, L., Vatavu, R.-D., \& Wobbrock, J. O. (2013). Understanding the Consistency of Users' Pen and Finger Stroke Gesture Articulation. Proceedings of Graphics Interface (p. to appear). Canadian Information Processing Society.

Anthony, L., \& Wobbrock, J. O. (2012). \$N-protractor: a fast and accurate multistroke recognizer. Proceedings of Graphics Interface (pp. 117-120). Canadian Information Processing Society.

Beery, K., Buktenica, N., \& Beery, N. A. (2004). The Beery-Buktenica Developmental Test of Visual-Motor Integration, 5th Edition. New Jersey: Modern Curriculum Press.

Brown, Q., \& Anthony, L. (2012). Toward Comparing the Touchscreen Interaction Patterns of Kids and Adults. Proceedings of the SIGCHI Workshop on Educational Software, Interfaces and Technology (p. 4pp).

Brown, Q., Hatley, L., Bonsignore, E. M., \& Druin, A. (2011). Mobile Natives: Unlocking the Potential of Educational Technology. ACM SIGCHI 2nd Workshop on UI Technologies and Their Impact on Educational Pedagogy (p. 4pp). 
Clawson, J., Lyons, K., Starner, T., \& Clarkson, E. (2005). The Impacts of Limited Visual Feedback on Mobile Text Entry for the Twiddler and Mini-QWERTY Keyboards. Proceedings of the IEEE International Symposium on Wearable Computers (pp. 170-177). IEEE Press. doi:10.1109/ISWC.2005.49

Crettez, J.-P. (1995). A set of handwriting families: style recognition. Proceedings of the International Conference on Document Analysis and Recognition (Vol. 1, pp. 489-494). IEEE Press. doi:10.1109/ICDAR.1995.599041

De Bruyn, D., \& Davis, A. (2008). Visual feedback is not essential for children to make the perpendicular bias. Perception ECVP Abstract Supplement, 37, 149.

Dean, A., \& Voss, D. (1999). Design and Analysis of Experiments. New York, NY, USA: Springer-Verlag.

Donker, A., \& Reitsma, P. (2007). Aiming and clicking in young children's use of the computer mouse. Computers in Human Behavior, 23(6), 2863-2874. doi:10.1016/j.chb.2006.06.002

Frisch, M., Heydekorn, J., \& Dachselt, R. (2009). Investigating multi-touch and pen gestures for diagram editing on interactive surfaces. Proceedings of the ACM International Conference on Interactive Tabletops and Surfaces (pp. 149-156). New York, New York, USA: ACM Press. doi:10.1145/1731903.1731933

Gustafson, S. (2012). Imaginary interfaces: touchscreen-like interaction without the screen. Extended Abstracts of the ACM SIGCHI Conference on Human Factors in Computing Systems (pp. 927-930). New York, New York, USA: ACM Press. doi:10.1145/2212776.2212867

Harris, A., Rick, J., Bonnett, V., Yuill, N., Fleck, R., Marshall, P., \& Rogers, Y. (2009). Around the Table: Are multiple-touch surfaces better than single-touch for children's collaborative interactions? In C. O'Malley, D. Suthers, P. Reimann, \& A. Dimitracopoulou (Eds.), Proceedings of the International Conference on Computer Supported Collaborative Learning (Vol. 4, pp. 335-344). ISLS.

Hinrichs, U., \& Carpendale, S. (2011). Gestures in the wild. Proceedings of the ACM SIGCHI Conference on Human Factors in Computing Systems (pp. 3023-3032). New York, New York, USA: ACM Press. doi:10.1145/1978942.1979391

Hourcade, J. P., Bederson, B. B., Druin, A., \& Guimbretière, F. (2004). Differences in pointing task performance between preschool children and adults using mice. ACM Transactions on Computer-Human Interaction, 11(4), 357-386. doi:10.1145/1035575.1035577

Inkpen, K. M. (2001). Drag-and-drop versus point-and-click mouse interaction styles for children. ACM ToCHI, 8(1), 1-33.

doi:10.1145/371127.371146

Joiner, R., Messer, D., Light, P., \& Littleton, K. (1998). It is best to point for young children: a comparison of children's pointing and dragging. Computers in Human Behavior, 14(3), 513-529. doi:10.1016/S0747-5632(98)00021-1

Jones, T. (1991). An Empirical Study of Children's Use of Computer Pointing Devices. Journal of Educational Computing Research, 7(1), 6176.

Kammer, D., Wojdziak, J., Keck, M., Groh, R., \& Taranko, S. (2010). Towards a formalization of multi-touch gestures. Proceedings of the ACM Conference on Designing Interactive Systems (pp. 49-58). New York, New York, USA: ACM Press. doi:10.1145/1936652.1936662

Kane, S. K., Wobbrock, J. O., \& Ladner, R. E. (2011). Usable gestures for blind people: understanding preference and performance. Proceedings of the ACM SIGCHI Conference on Human Factors in Computing Systems (pp. 413-422). ACM Press.

Kratz, S., \& Ballagas, R. (2009). Unravelling seams: improvoing mobile gesture recognition with visual feedback techniques. Proceedings of the ACM SIGCHI Conference on Human Factors in Computing Systems (pp. 937-940). ACM Press.

Li, Y. (2010). Gesture search: a tool for fast mobile data access. Proceedings of the ACM Symposium on User Interface Software and Technology (pp. 87-96). ACM Press.

Lin, E., Cassidy, A., Hook, D., \& Baliga, A. (2002). Hand tracking using spatial gesture modeling and visual feedback for a virtual DJ system. Proceedings of the IEEE International Conference on Multimodal Interfaces (pp. 197-202). IEEE Press. doi:10.1109/ICMI.2002.1166992

Long, A. C., Landay, J. A., Rowe, L. A., \& Michiels, J. (2000). Visual similarity of pen gestures. Proceedings of the ACM SIGCHI Conference on Human Factors in Computing Systems (pp. 360-367). New York, New York, USA: ACM Press. doi:10.1145/332040.332458

Morris, M. R., Wobbrock, J. O., \& Wilson, A. D. (2010). Understanding users' preferences for surface gestures. Proceedings of Graphics Interface (pp. 261-268). Canadian Information Processing Society.

Piaget, J. (1983). Piaget's Theory. In P. Mussen (Ed.), Handbook of Child Psychology (4th ed.). New York, NY, USA: Wiley \& Sons. 
Read, J. C., MacFarlane, S., \& Casey, C. (2002). Pens behaving badly-usability of pens and graphics tablets for text entry with children. Adjunct Proceedings of the ACM Symposium on User Interface Software and Technology (Vol. 2, pp. 21-22). Paris, France: ACM Press. doi:10.1145/572020.572026

Rick, J., Harris, A., Marshall, P., Fleck, R., Yuill, N., \& Rogers, Y. (2009). Children designing together on a multi-touch tabletop: An analysis of spatial orientation and user interactions. Proceedings of the International Conference on Interaction Design and Children (pp. 106114). New York, NY, USA: ACM Press. doi:http://doi.acm.org/10.1145/1551788.1551807

Rösblad, B. (2006). Reaching and eye-hand coordination. In A. Henderson \& C. Pehoski (Eds.), Hand Function in the Child: Foundations for Remediation. St. Louis, MO, USA: Mosby Elsevier.

Rubine, D. (1991). Specifying gestures by example. SIGGRAPH Computer Graphics, 25(4), 329-337. doi:http://doi.acm.org/10.1145/127719.122753

Ryall, K., Morris, M. R., Everitt, K., Forlines, C., \& Shen, C. (2006). Experiences with and observations of direct-touch tabletops. Proceedings of the IEEE International Workshop on Horizontal Interactive Human-Computer Systems (p. 8pp). IEEE Press. doi:10.1109/TABLETOP.2006.12

Schmidt, M., \& Weber, G. (2010). Enhancing Single Touch Gesture Classifiers to Multitouch Support. In K. Miesenberger, J. Klaus, W. Zagler, \& A. Karshmer (Eds.), Proceedings of the International Conference on Computers Helping People with Special Needs (Vol. 6180, pp. 490-497). Berlin, Heidelberg: Springer Berlin Heidelberg. doi:10.1007/978-3-642-14100-3

Shuler, C. (2009). Pockets of Potential: Using Mobile Technologies to Promote Children's Learning (p. 56pp). New York, NY: Joan Ganz Cooney Center at Sesame Workshop.

Smyth, M. M. (1989). Visual control of movement patterns and the grammar of action. Acta psychologica, 70(3), $253-265$.

Srihari, S. N., Cha, S.-H., Arora, H., \& Lee, S. (2001). Individuality of Handwriting: A Validation Study. Proceedings of the International Conference on Document Analysis and Recognition (p. 106109). IEEE Press.

Tu, H., Ren, X., \& Zhai, S. (2012). A comparative evaluation of finger and pen stroke gestures. Proceedings of the ACM SIGCHI Conference on Human Factors in Computing Systems (pp. 1287-1296). New York, New York, USA: ACM Press. doi:10.1145/2207676.2208584

Vatavu, R.-D., Anthony, L., \& Wobbrock, J. O. (2012). Gestures as point clouds: a \$P recognizer for user interface prototypes. Proceedings of the ACM International Conference on Multimodal Interaction (pp. 273-280). New York, New York, USA: ACM Press. doi:10.1145/2388676.2388732

Vinter, A., \& Meulenbroek, R. (1993). The role of manual dominance and visual feedback in circular drawing movements. Journal of Human Movement Studies, 25, 11-37.

Wobbrock, J. O., Morris, M. R., \& Wilson, A. D. (2009). User-defined gestures for surface computing. Proceedings of the ACM SIGCHI Conference on Human Factors in Computing Systems (pp. 1083-1092). New York, New York, USA: ACM Press. doi:10.1145/1518701.1518866

Zhai, S., Kristensson, P. O., Appert, C., Anderson, T. H., \& Cao, X. (2012). Foundational Issues in Touch-Surface Stroke Gesture Design - An Integrative Review. Foundations and Trends in Human-Computer Interaction, 5(2), 97-205.

Zhao, S., Dragicevic, P., Chignell, M., Balakrishnan, R., \& Baudisch, P. (2007). Earpod: eyes-free menu selection using touch input and reactive audio feedback. Proceedings of the ACM SIGCHI Conference on Human Factors in Computing Systems (pp. 1395-1404). New York, New York, USA: ACM Press. doi:10.1145/1240624.1240836 